\title{
Associação entre o risco da ocorrência da síndrome da apneia obstrutiva do sono e o sexo, o índice de massa corporal e a ocorrência de ronco em estudantes de Medicina
}

Association between the risk of obstructive sleep apnea syndrome with gender, body mass index, and snoring occurrence in Medical students

Asociación entre el riesgo de ocurrencia del síndrome de apnea obstructiva del sueño y el sexo, el índice de masa corporal y la ocurrencia de ronquidos en estudiantes Médicos

ORCID: https://orcid.org/0000-0001-5578-9641 Universidade Federal de Alfenas, Brasil

E-mail: luigi.peloso@sou.unifal-mg.edu.br Sandra Pereira Zanon Alves

ORCID: https://orcid.org/0000-0002-3464-3819 Universidade Federal de Alfenas, Brasil

E-mail: sandra.alves@ @ou.unifal-mg.edu.br

Rogério Martins Pavesi Gonçalves ORCID: https://orcid.org/0000-0001-7151-4744 Universidade Federal de Alfenas, Brasil E-mail: rogerio.goncalves@sou.unifal-mg.edu.br

Marcus Augustus Alves Ferreira ORCID: https://orcid.org/0000-0002-3381-3986 Universidade Federal de Alfenas, Brasil E-mail: marcusufff@yahoo.com.br

Sara Regina Alcalde Domingos ORCID: https://orcid.org/0000-0001-8532-4116 Universidade Federal de Alfenas, Brasil E-mail: srad19@hotmail.com

Carlos Bernardo Assis Trovão ORCID: https://orcid.org/0000-0002-8982-5767 Universidade Federal de Alfenas, Brasil E-mail: trovaobernardo@gmail.com José Antônio Dias Garcia ORCID: https://orcid.org/0000-0002-4024-3045 Universidade José do Rosário Vellano, Brasil E-mail: jadiasgarcia@gmail.com

Gema Galgani de Mesquita Duarte ORCID: https://orcid.org/0000-0002-4899-5479 Universidade Federal de Alfenas, Brasil

E-mail: gema.mesquita@unifal-mg.edu.br

Edvaldo José Rodrigues Cardoso

ORCID: https://orcid.org/0000-0002-9701-450X Universidade Federal de Alfenas, Brasil

E-mail: edvaldo.cardoso@unifal-mg.edu.br Adriana Dias

ORCID: https://orcid.org/0000-0002-2883-431X Universidade Federal de Alfenas, Brasil E-mail: adriana.dias@unifal-mg.edu.br Evelise Aline Soares

ORCID: https://orcid.org/0000-0001-7838-687X Universidade Federal de Alfenas, Brasil E-mail: evelise.anatomia@gmail.com

\section{Resumo}

Introdução: O sono entre os estudantes de medicina tem sido um fator de discussão na comunidade acadêmica, visto que a mudança de vida ao ingressar no ensino superior e o alto nível de cobrança do curso contribuem para prejuízos na qualidade do sono com uma alta incidência de distúrbios, como a Síndrome da Apneia Obstrutiva do Sono (SAOS). Objetivo: Avaliar a associação entre o risco da ocorrência da síndrome da apneia obstrutiva do sono e o sexo, o índice de massa corporal (IMC) e a ocorrência de ronco em estudantes do terceiro período do curso de graduação em medicina da Universidade Federal de Alfenas. Metodologia: Fizeram parte da amostra estudantes do curso de medicina que responderam a dois instrumentos de coleta de dados: I) Questionário Sociodemográfico e II) 
Questionário de Berlim, aplicados pelos pesquisadores no início do terceiro período do curso. Resultados: Observouse maior frequência de ronco entre os estudantes com alto risco para SAOS em relação aos de baixo risco, porém quando relacionados sexo e IMC com a SAOS não foram encontrados valores estatísticos significativos para classificá-los como fatores preditivos ou não para a ocorrência da síndrome. Conclusão: Apesar de não terem sido encontrados valores estatísticos significativos em relação ao sexo e ao IMC, os estudantes apresentaram distúrbios relacionados ao sono e valores absolutos de ocorrência de risco para SAOS, fatores que podem prejudicá-los no desempenho acadêmico.

Palavras-chave: Sono; Estudantes; Medicina; Apneia obstrutiva do sono.

\begin{abstract}
Introduction: Sleep among medical students has been a factor of discussion in the academic community. Life changes when entering higher education and higher education requirements contribute to losses in the quality of sleep together with a greater incidence of disorders such as Obstructive Sleep Apnea Syndrome. Objective: To evaluate the risk of obstructive sleep apnea syndrome with gender, BMI, and snoring occurrence in third-year medical students at the Universidade Federal de Alfenas. Methodology: The sample consisted of medical students who answered two data collection tools: I) Sociodemographic Questionnaire and II) Berlin Questionnaire. Researchers applied both at the beginning of the third-period course. Results: Higher frequency of snoring among students at high risk for OSAS was observed compared to those at low risk. However, when relating gender and BMI to OSAS, values statistically significant have not been found to classify them as predictive or not predictive factors for the syndrome occurrence. Conclusion: Although significant values for gender and BMI have not been found, the students presented sleep disorders and absolute values of risk occurrence for OSAS. Factors that may affect their academic performance.
\end{abstract}

Keywords: Sleep; Students; Medicine; Obstructive sleep apnea.

\begin{abstract}
Resumen
Introducción: El sueño entre los estudiantes de medicina ha sido un factor de discusión en la comunidad académica, ya que el cambio de vida al ingresar a la educación superior y el alto nivel de carga por el curso contribuyen a dañar la calidad del sueño con una alta incidencia de trastornos como como el Síndrome de Apnea Obstructiva del Sueño. Objetivo: Evaluar la asociación entre el riesgo de ocurrencia de síndrome de apnea obstructiva del sueño y sexo, IMC y ocurrencia de ronquidos en estudiantes de tercer período de medicina de la Universidad Federal de Alfenas. Metodología: Los estudiantes de Medicina que respondieron a dos instrumentos de recolección de datos fueron parte de la muestra: I) Cuestionario Sociodemográfico y II) Cuestionario de Berlín, aplicado por los investigadores al inicio del tercer período del curso. Resultados: Se observó una mayor frecuencia de ronquidos entre los estudiantes con alto riesgo de SAOS en comparación con los de bajo riesgo, sin embargo, cuando el sexo y el IMC se relacionaron con SAOS, no se encontraron valores estadísticamente significativos para clasificarlos como factores predictivos o no para la aparición del síndrome. Conclusión: Aunque no se encontraron valores estadísticamente significativos en relación al género e IMC, los estudiantes presentaron trastornos relacionados con el sueño y valores absolutos para la ocurrencia de riesgo de SAOS, factores que pueden afectarlos en su desempeño académico.
\end{abstract}

Palabras clave: Sueño; Estudiantes; Medicina; Apnea obstructiva del sueño.

\title{
1. Introdução
}

O sono tem sido um fator de discussão na comunidade acadêmica, sendo notório que o ingresso em uma universidade gera grandes impactos na vida dos estudantes de medicina, já que a mudança na rotina, o novo nível de cobrança e as responsabilidades da futura profissão, podem causar impactos na saúde e na qualidade do sono (Preišegolavičiūtė et al., 2010; Ferreira et al., 2020). Estudos realizados por Corrêa et al (2017), em um grupo de 540 estudantes de medicina evidenciaram que a má qualidade do sono se mostrou elevada, principalmente entre os alunos dos anos iniciais.

A ação do sono está relacionada a processos fisiológicos e neuro-cognitivos de extrema importância e a sua privação, em especial por estudantes, pode estar relacionada à dificuldade de concentração, à diminuição da atenção, à alteração na capacidade psicomotora e ao baixo rendimento escolar, em destaque a diminuição na capacidade de resolver problemas, o aumento nas taxas de erro e a redução da competência de reter informações e interpretar exames, ação comum para os estudantes de medicina (Juliano, 2013).

Um importante distúrbio do sono que apresenta elevado risco de incidência e se torna um fator preocupante para os estudantes de medicina é a Síndrome da Apneia Obstrutiva do Sono (SAOS), visto que esse grupo é submetido diariamente a uma elevada carga horária, responsabilidades e uma grande gama de atividades (Ribeiro et al., 2014). A SAOS é caracterizada 
pela ocorrência de eventos de obstrução parcial das vias aéreas superiores, denominada hipopneia, ou de obstrução total, caracterizando a apneia propriamente dita. Como resultado ela afeta a qualidade de vida dos portadores dessa síndrome, sendo uma morbidade que prejudica todo o cotidiano (Mediano et al., 2007). Pesquisas relacionando a qualidade de sono e a SAOS, investigaram ronco e apneia em um grupo de universitários e de acordo com Saygin (2016) 3\% deles apresentavam ronco e 1,5\% apneia, já no estudo de Akintude, Salawu e Opadijo (2017) detectou-se que 44,2\% dos estudantes apresentavam ronco e 30,6\% SAOS (Saygin et al., 2016; Akintunde et al., 2014).

O presente estudo tem por objetivo avaliar a associação entre o risco da ocorrência da síndrome da apneia obstrutiva do sono e o sexo, o IMC e a ocorrência de ronco em estudantes do terceiro período do curso de graduação em medicina da Universidade Federal de Alfenas (UNIFAL- MG).

\section{Metodologia}

Aspectos éticos - O estudo foi aprovado pelo Comitê de Ética em Pesquisa com seres humanos da UNIFAL-MG, sob parecer 1.623.096, todos os participantes do estudo estavam de acordo com a pesquisa e assinaram o Termo de Consentimento Livre e Esclarecido.

Amostra - Foi realizado um estudo de coorte transversal por meio do levantamento de dados primários para a avaliação do perfil da qualidade de sono entre universitários do curso de medicina. Para tal, foram utilizados instrumentos de coleta de dados padronizados e validados para a população brasileira, os quais foram distribuídos e respondidos em sala de aula, por 46 alunos do curso de medicina da Universidade Federal de Alfenas, no município de Alfenas, Minas Gerais, Brasil.

Instrumentos de avaliação - Os instrumentos de coleta de dados utilizados foram questionários validados e reconhecidos internacionalmente, sendo eles:

1. Questionário Sociodemográfico: Foi utilizada uma adaptação da seção A do Composite International Development Interview (CIDI), versão 2.1, para a identificação das seguintes variáveis sociodemográficas: idade, peso, estatura, sexo, estado civil, cor/etnia, atividade profissional e de lazer, doenças, internações, uso de medicamentos, tabagismo, etilismo, e percepção de saúde autorrelatada (Quintana et al., 2007).

2. Questionário de Berlim (Berlim Questionnaire - BQ): Elaborado no ano de 1996 durante a Conference on Sleep in Primary Care, conferência realizada em Berlim com a participação de 120 médicos pulmonares e de atenção primária dos EUA e da Alemanha, e validado por Netzer e colaboradores em 1999, o Questionário de Berlim tem o intuito de delimitar o risco para apneia do sono, na atenção primária, através de uma série de perguntas para detectar a presença dos fatores ou comportamentos de riscos mais comuns para a ocorrência da síndrome da apneia obstrutiva do sono (SAOS). A determinação do alto ou baixo risco para a SAOS é baseada nas respostas dadas em cada categoria de itens, sendo que as categorias 1 e 2 são positivas quando o respondente apresenta duas ou mais respostas positivas dentre as questões de cada uma dessas categorias, já a categoria 3 é considerada positiva quando o respondente apresenta histórico de hipertensão arterial sistêmica (HAS) ou índice de massa corporal $(\mathrm{IMC})>30 \mathrm{~kg} / \mathrm{m} 2$. Assim, a positividade em duas ou três categorias define uma pontuação ou score de alto risco para a SAOS e a positividade em apenas uma ou em nenhuma categoria define uma pontuação ou score de baixo risco (Netzer et al., 1999; Vaz et al., 2011). Este questionário inclui 10 itens, organizados em 3 categorias referentes à roncopatia e apneias presenciadas (5 itens), sonolência diurna (4 itens) e hipertensão arterial/obesidade (1 item). Informações sobre gênero, idade, altura, peso, circunferência do pescoço e etnia também são solicitadas. No Brasil, o Questionário de Berlim, atualmente, 
é a principal ferramenta para priorizar os pacientes com maiores fatores de risco associados à SAOS, uma vez que o exame padrão ouro, a polissonografia, tem um custo elevado e não é amplamente disponibilizado pelo Sistema Único de Saúde (Melo et al., 2016).

Análise e apresentação dos dados - Os resultados serão apresentados de forma descritiva através de tabelas comparativas junto a discussões baseadas em literaturas, segundo o modelo de estudo do tipo quali-quanti (Pereira et al., 2018).

Tratamento estatístico - O teste Exato de Fisher foi utilizado para comparar as variáveis sexo, IMC e ocorrência de ronco com o risco para a ocorrência da síndrome da apneia obstrutiva do sono. As análises estatísticas foram realizadas no Programa R (R Core Team, 2020) adotando-se o nível de significância de 5\%.

\section{Resultados e Discussão}

Participaram da amostra, 46 estudantes, 76,67\% do número de matriculados. Os outros 14 estudantes, ou se recusaram a responder ou não estavam na sala de aula no momento em que os instrumentos foram aplicados.

A amostragem revelou que os estudantes tinham média de idade de 21,45 anos $( \pm 3,69$ anos), dos quais 19 identificavam-se como homens $(41,30 \%)$ e 27 como mulheres (58,70\%). Com relação à cor/etnia 80,00\% são autodeclarados brancos, $20,00 \%$ pardos e nenhum se autodeclarou preto, amarelo ou indígena. Apenas um $(2,17 \%)$ estudante era natural de Alfenas-MG. No perfil residencial apenas 3 (6,52\%) estudantes residiam com os pais, 39 (84,78\%) residiam em moradias compartilhadas/repúblicas e $4(6,52 \%)$ em pensionatos estudantis. O valor médio calculado do IMC foi de $22,51 \mathrm{~kg} / \mathrm{m} 2$ $( \pm 3,27)$, desses $10,87 \%$ apresentaram IMC abaixo de $18,53 \mathrm{~kg} / \mathrm{m} 2$ (abaixo do peso), $69,57 \%$ apresentaram IMC entre 18,5 e $24,9 \mathrm{~kg} / \mathrm{m} 2$ (peso ideal), $15,22 \%$ apresentaram IMC entre 25 e $29,9 \mathrm{~kg} / \mathrm{m} 2$ (sobrepeso) e apenas 2 pessoas $(4,35 \%)$ tinham IMC acima de $30 \mathrm{~kg} / \mathrm{m} 2$ (obesidade). Em relação ao estado civil, a grande maioria (97,83\%) é solteiro, enquanto apenas um estudante é casado (2,17\%). Na autopercepção em saúde durante os últimos cinco anos 50,00\% dos alunos classificaram sua saúde como excelente, $23,91 \%$ como muito boa, $15,22 \%$ como boa, $8,70 \%$ como regular e apenas $2,17 \%$ como ruim.

Na Tabela 1 estão apresentadas as características sociodemográficas e físicas dos estudantes participantes de acordo com as categorias do Questionário de Berlim. 
Tabela 1 - Características sociodemográficas e físicas dos estudantes participantes de acordo com as categorias do Questionário de Berlim.

\begin{tabular}{|c|c|c|c|c|c|c|}
\hline \multirow[t]{2}{*}{ Questionário de Berlim } & \multicolumn{2}{|c|}{ Categoria 1} & \multicolumn{2}{|c|}{ Categoria 2} & \multicolumn{2}{|c|}{ Categoria 3} \\
\hline & Negativo & Positivo & Negativo & Positivo & Negativo & Positivo \\
\hline & \multicolumn{6}{|c|}{ Número de participantes } \\
\hline \multirow[t]{2}{*}{$\mathrm{n}$} & 40 & 6 & 38 & 8 & 44 & 2 \\
\hline & \multicolumn{6}{|c|}{ Sexo } \\
\hline Feminino & 25 & 2 & 22 & 5 & 27 & 0 \\
\hline \multirow[t]{2}{*}{ Masculino } & 15 & 4 & 16 & 3 & 17 & 2 \\
\hline & \multicolumn{6}{|c|}{ Estado civil } \\
\hline Casado & 0 & 1 & 1 & 0 & 1 & 0 \\
\hline Divorciado & 1 & 0 & 1 & 0 & 1 & 0 \\
\hline \multirow[t]{2}{*}{ Solteiro } & 39 & 5 & 36 & 8 & 42 & 2 \\
\hline & \multicolumn{6}{|c|}{ Cor/etnia } \\
\hline Branca & 32 & 4 & 30 & 6 & 35 & 1 \\
\hline \multirow[t]{2}{*}{ Parda } & 7 & 2 & 7 & 2 & 8 & 1 \\
\hline & \multicolumn{6}{|c|}{ Tipo de moradia } \\
\hline Com os pais & 2 & 1 & 3 & 0 & 3 & 0 \\
\hline Pensão & 4 & 0 & 3 & 1 & 4 & 0 \\
\hline \multirow[t]{2}{*}{ República } & 34 & 5 & 32 & 7 & 37 & 2 \\
\hline & \multicolumn{6}{|c|}{$\mathrm{IMC}\left(\mathrm{em} \mathrm{kg} / \mathrm{m}^{2}\right)$} \\
\hline Média \pm desvio padrão & $22,27 \pm 3,14$ & $24,12 \pm 3,97$ & $22,29 \pm 3,30$ & $23,54 \pm 3,11$ & $22,14 \pm 2,82$ & $30,60 \pm 0,50$ \\
\hline \multirow[t]{2}{*}{ Acima de $24 \mathrm{~kg} / \mathrm{m}^{2}$} & $25,85 \pm 1,90$ & $28,52 \pm 2,45$ & $26,73 \pm 2,42$ & $25,33 \pm 1,08$ & $25,50 \pm 1,09$ & $30,60 \pm 0,50$ \\
\hline & \multicolumn{6}{|c|}{ Idade (em anos) } \\
\hline Média \pm desvio padrão & $21,45 \pm 3,69$ & $23 \pm 4,52$ & $21,79 \pm 4,09$ & $21,00 \pm 1,85$ & $21,48 \pm 3,71$ & $25,5 \pm 4,95$ \\
\hline
\end{tabular}

Fonte: Elaborado pelos autores com base nos dados da pesquisa.

Na categoria I do Questionário de Berlim, é avaliada a presença e a qualidade do ronco. O ronco primário é o primeiro indicador de SAOS grave, e tem uma sensibilidade de $82,6 \%$ e uma especificidade de $43 \%$ para identificar a SAOS, dessa forma se faz necessário associar outros elementos para melhor caracterização da síndrome (Associação Brasileira de Otorrinolaringologia e Cirurgia Cérvico-Facial et al., 2012).

No total, seis $(13,04 \%)$ estudantes responderam que roncam durante a noite, $31(67,39 \%)$ que não roncam e nove $(19,56 \%)$ que não sabem. Entre os seis que roncam, quatro $(66,66 \%)$ são homens e dois $(33,33 \%)$ são mulheres. Dos que roncam, três $(50,00 \%)$ assinalaram que roncam ligeiramente mais alto que a respiração, um (16,66\%) que ronca tão alto que pode ser ouvido em diversos cômodos da casa e dois $(33,33 \%)$ roncam na mesma altura que a fala humana. Em relação a frequência de ronco, quatro $(66,66 \%)$ roncam todos os dias da semana, um $(16,66 \%)$ ronca de três a quatro vezes por semana, assim como, um $(16,66 \%)$ ronca de uma a duas vezes na semana. Quatro $(66,66 \%)$ participantes responderam que o ronco incomoda outras pessoas, enquanto dois (33,33\%) responderam que não. Um participante $(16,66 \%)$ respondeu que alguém já percebeu que há perda da respiração durante a noite de três a quatro vezes na semana, dois $(33,33 \%)$ que isso ocorre de uma a duas vezes na semana e metade $(50,00 \%)$ respondeu que esse evento nunca ou quase nunca ocorre. Como apresentado na 
Tabela 1, a incidência de ronco é proporcionalmente maior no sexo masculino, numa relação de aproximadamente 2,9:1 em relação ao sexo feminino. Esta informação está de acordo com a pesquisa realizada no Laboratório do Sono do Hospital Português da Bahia, na qual a prevalência de ronco é de 71,7\% nos homens e de 28,3\% nas mulheres, prevalecendo uma proporção de 2,8:1 para a população geral (Peppard et al., 2013). Com relação ao ronco, conforme tabela 2, identificou-se maior frequência entre estudantes de alto risco para SAOS quando comparados aos de baixo risco $(p=0,0013)$. Dos 46 participantes avaliados, $6(13,04 \%)$ apresentaram a categoria I positiva para risco de SAOS.

Na categoria II, dois $(4,34 \%)$ estudantes responderam que se sentem fatigados após uma noite de sono todos os dias, seis $(13,04 \%)$ de três a quatro vezes na semana, dezessete $(36,95 \%)$ de uma a duas vezes na semana, quatro $(8,69 \%)$ de uma a duas vezes ao mês e quinze $(32,60 \%)$ nunca ou quase nunca. Sete $(15,21 \%)$ estudantes assinalaram que quase todos os dias se sentem fatigados e/ou cansados durante o dia, nove (19,56\%) de três a quatro vezes por semana, oito (17,39\%) de uma a duas vezes por semana, seis $(13,04 \%)$ de uma a duas vezes ao mês e quatorze $(30,43 \%)$ nunca ou quase nunca se sentem cansados. Nenhum $(0,00 \%)$ participante revelou ter cochilado ou caído no sono enquanto dirigia. Dessa maneira, dos 46 entrevistados para a categoria II, dois $(4,34 \%)$ estudantes não responderam às perguntas e oito $(17,39 \%)$ obtiveram resultado positivo. Como pode ser observado na Tabela 1, dentre os estudantes positivados na categoria II e que com isso apresentam um maior risco para a SAOS, sete $(87,5 \%)$ residiam em repúblicas, um $(12,50 \%)$ em pensão e nenhum $(0,00 \%)$ morava com os pais, resultado que está de acordo com literaturas ao sugerir a influência que mudanças de hábitos de vida, como não morar mais com os pais, têm sobre a qualidade do sono dos estudantes (Ferreira et al., 2020).

Na categoria III do Questionário de Berlim é avaliado a presença de hipertensão e o valor do IMC, sendo que ela é positiva quando o paciente tem hipertensão ou IMC $>30 \mathrm{~kg} / \mathrm{m} 2$. Um alto índice de IMC representa um importante preditivo para a ocorrência da SAOS, visto que a obesidade, caracterizada por IMC $>30 \mathrm{~kg} / \mathrm{m} 2$, está presente em cerca de $70 \%$ dos pacientes com essa síndrome e destaca-se por ser um fator reversível (Malhotra \& White, 2002). Neste estudo, dentre os 46 estudantes avaliados, apenas dois (4,34\%) apresentaram positividade para essa categoria por possuírem IMC>30 kg/ m2 e, em relação à presença de HAS, nenhum $(0,00 \%)$ estudante afirmou possuir essa condição, um $(2,17 \%)$ alegou não saber responder e três $(6,52 \%)$ não responderam. Ao final, 3 estudantes foram classificados com alto risco para a SAOS, dos quais um foi avaliado com peso normal (IMC $=23,84 \mathrm{~kg} / \mathrm{m} 2)$, um com sobrepeso $(\mathrm{IMC}=26,79 \mathrm{~kg} / \mathrm{m} 2)$ e um como obeso $(\mathrm{IMC}=30,25$ $\mathrm{kg} / \mathrm{m} 2$ ), conforme apresentado na Tabela 2.

Na Tabela 2 estão apresentados os resultados do teste Exato de Fisher (valores-p) obtidos na comparação das variáveis sexo, IMC e ocorrência de ronco com o risco para a ocorrência da síndrome da apneia obstrutiva do sono. 
Tabela 2 - Classificação do risco para SAOS dos estudantes participantes segundo sexo, IMC e ronco e resultado do teste exato de Fisher

\begin{tabular}{cccc}
\hline \multirow{2}{*}{ Demais variáveis } & SAOS & Alto & Valor-p \\
\cline { 2 - 4 } & Baixo & 0 & 0,9999 \\
\hline Sexo & 27 & 3 & 0,0978 \\
\hline Feminino & 16 & & \\
\hline Masculino & 51 & 1 & 0,0013 \\
\hline IMC & 5 & 1 & \\
\hline Baixo peso & 6 & 1 & \\
\hline Adequado & 1 & & \\
\hline Sobrepeso & & 0 & \\
\hline Obesidade & 9 & 0 & \\
\hline Ronco & 31 & 3 & \\
\hline Não sei & 3 & & \\
\hline Não & & & \\
\hline Sim & & & \\
\hline
\end{tabular}

Fonte: Elaborado pelos autores com base nos dados da pesquisa.

No presente estudo, embora ocorra predominância para estudantes do sexo masculino com indicativos de risco de SAOS, não houve diferença estatística quando comparado os sexos (valor-p = 0,9999) conforme apresentado na Tabela 2. Dessa forma temos uma contradição com os estudos de Koo, nos quais afirma-se que a SAOS acontece com maior frequência em mulheres na pós-menopausa, por volta dos 45 anos, do que em mulheres na pré-menopausa (Koo et al., 2017). A progesterona tem grande influência na função respiratória, mulheres na pré-menopausa têm níveis maiores de progesterona circulante, fator que possui um efeito inibitório sobre o fechamento das vias respiratórias, reduzindo a colapsibilidade do músculo genioglosso (Vagiakis et al., 2006).

Ao se comparar o risco para a ocorrência da síndrome da apneia obstrutiva do sono com o IMC, observa-se que não há diferença estatística $(\mathrm{p}=0,0978)$ entre as diferentes classificações de peso, ou seja, há semelhança entre as variadas classificações de peso na classificação dos riscos para SAOS.

Grande parte das literaturas sugere forte relação entre o IMC elevado e a SAOS, em estudo realizado por Modena foi constatado que a obesidade é um dos principais fatores de risco para a SAOS, sendo 80,34\% dos entrevistados, que apresentavam obesidade, com alto risco para o desenvolvimento da SAOS contra 19,66\% apresentando baixo risco (Modena et al, 2017). Entretanto, no estudo de Gonzaga foi encontrada baixa relação estatística entre IMC e a ocorrência da SAOS, fato que vai de encontro ao que foi obtido neste estudo, tendo em vista as características do grupo de estudantes entrevistados (Gonzaga et al, 2019).

Para Pinto et al., 2011 existem controvérsias em relação aos parâmetros preditivos mais relevantes da obesidade que levam à ocorrência da SAOS. Em seu estudo foi observado que dentre as medidas antropométricas de classificação da obesidade como IMC, circunferência do pescoço, circunferência do quadril, circunferência abdominal e índice de Mallampati modificado, o mais significativo para associação com a SAOS é a circunferência do pescoço. 


\section{Considerações Finais}

É notório que a qualidade de sono é crucial para os estudantes universitários, principalmente para os da área da medicina que têm elevada carga horária e grandes responsabilidades (BENEVANTE et al., 2014). Por isso, os fatores predisponentes da SAOS devem se manter como objeto de estudos no mundo acadêmico e devem permanecer como sinal de alerta para o bem-estar dos estudantes.

No presente estudo, houve associação significativa (valor-p $<0,05$ ) entre o risco de ocorrência da SAOS e a ocorrência de ronco, mas não houve associação significativa (valor-p > 0,05) entre o risco de ocorrência da SAOS e o sexo e nem com o IMC.

Apesar de neste estudo não terem sido encontrados valores estatísticos significativos que comprovem a relação entre o sexo masculino e o IMC elevado como determinantes para um alto risco de ocorrência da SAOS, os estudantes que responderam aos questionários apresentaram distúrbios do sono importantes e que devem ser analisados.

Por fim, é importante ressaltar o baixo número de estudantes pertencentes a amostra e a variação do número de respostas obtidas em cada pergunta do BQ, fatores que podem ter influenciado o resultado obtido ao final do estudo.

Assim, para trabalhos futuros, sugere-se aumentar o número de estudantes pertencentes ao estudo, aplicando os questionários em mais de uma turma e em períodos distintos do curso de medicina, afim de ampliar o número de respostas em cada questionário e analisar os diferentes resultados obtidos ao fim dos estudos.

\section{Referências}

Akintunde, A. A., Salawu, A. A., \& Opadijo, O. G. (2014). Assessment of Snoring and obstructive sleep apnoea in a Nigerian university: Association with cardiovascular risk factors. Nigerian Medical Journal, 55(6), 469-473. https://doi.org/10.4103/0300-1652.144698

Associação Brasileira de Otorrinolaringologia e Cirurgia Cérvico-Facial, Academia Brasileira de Neurologia, Sociedade Brasileira de Cardiologia, Sociedade Brasileira de Pediatria, \& Sociedade Brasileira de Pneumologia e Tisiologia. (2012). Obstructive sleep apnea and primary snoring: diagnosis. Brazilian Journal of Otorhinolaryngology, 80(1), S1-S16. https://doi.org/10.5935/1808-8694.2014S001

Benavente, S. B. T., Silva, R. M. d., Higashi, A. B., Guido, L. d. A., \& Costa, A. L. S. (2014). Influence Of Stress Factors And Socio-demographic Characteristics On The Sleep Quality Of Nursing Students. Journal of the Nursing School of the University of Sao Paulo, 48(03). https://doi.org/10.1590/S0080-623420140000300018

Corrêa, C. d. C., Oliveira, F. K. d., Pizzamiglio, D. S., Ortolan, E. V. P., \& Weber, S. A. T. (2017). Sleep quality in medical students: a comparison across the various phases of the medical course. Jornal Brasileiro de Pneumologia, 43(04), 285-289. https://doi.org/10.1590/S1806-37562016000000178

Ferreira, M. A. A., Domingos, S. R. A. ., Trovão, C. B. A. ., Domiciano, D. C. ., Silva , B. A. da ., Rocha , L. G. ., Carmello, L. do M., \& Soares, E. A. . (2020). Sleep Quality Profile of a group of Medical Students. Research, Society and Development, 9(9), e718997806. https://doi.org/10.33448/rsd-v9i9.7806

Gonzaga, D. B., Cavalcante, E. R. B., Souza, N. D. d., Barbosa, I. D., \& Feitoza, C. C. (2020). Snoring, obstructive sleep apnea syndrome and body mass index in the university environment. Revista Enfermagem Digital Cuidado e Promoção da Saúde, 5(1), 3-7. http://www.dx.doi.org/10.5935/24465682.20200002

Juliano, M. L. (2013). Importância da Qualidade do Sono em Universitários e Médicos Residentes. Revista Neurociências, 21(3), 331-332. https://doi.org/10.34024/rnc.2013.v21.8154

Koo, S. K., Ahn, G. Y., Choi, J. W., Kim, Y. J., Jung, S. H., Moon, J. S., \& Lee, Y. I. (2017). Obstructive sleep apnea in postmenopausal women: a comparative study using drug induced sleep endoscopy. Brazilian Journal of Otorhinolaryngology, 83(3), 285-291. https://doi.org/10.1016/j.bjorl.2016.03.011

Malhotra, A., \& White, D. P. (2002, July 20). Obstructive sleep apnoea. The Lancet, 360(9328), 237-245. https://doi.org/10.1016/S0140-6736(02)09464-3

Mediano, O., Barceló, A., Peña, M. d. L., Gozal, D., Augusti, A., \& Barbé, F. (2007, January 30). Hipersonolência diurna e va riáveis polissonográficas em doentes com síndroma de apneia do sono. Revista Portuguesa de Pneumologia, 8(6), 896-898. https://doi.org/10.1016/S2173-5115(07)70384-6

Melo, M. H. A., Neves, D. D., Ferreira, L. V. M. V., Moreira, M. L. V., Nigri, R., \& Simões, S. M. G. (2016). Questionários e Escalas úteis na pesquisa da Síndrome da Apneia Obstrutiva do Sono. Revista Hospital Universitário Pedro Ernesto, 15(1). https://doi.org/10.12957/rhupe.2016.22368.

Modena, D. A. O., Cazzo, E., Cândido, E. C., Baltieri, L., Silveira, L. J. B. d., Almeida, A. M. N. d., Gobato, R. C., \& Chaim, E. A. (2017). Síndrome da apneia obstrutiva do sono em indivíduos portadores de obesidade: um estudo transversal. Revista da Associação Médica Brasileira, 63(10), 862-868. https://doi.org/10.1590/1806-9282.63.10.862

Netzer NC, Stoohs RA, Netzer CM, et al. Using the Berlin Questionnaire to identify patients at risk for the sleep apnea syndrome. Ann Intern Med. 1999; 131(7):485-491. http://dx.doi. org/10.7326/0003-4819-131-7-199910050-00002

Peppard, P. E., Young, T., Barnet, J. H., Palta, M., Hagen, E. W., \& Hla, K. M. (2013). Increased Prevalence of Sleep-Disordered Breathing in Adults. American Journal of Epidemiology, 117(9), 1006-1014. https://doi.org/10.1093/aje/kws342 
Research, Society and Development, v. 10, n. 10, e544101019281, 2021

(CC BY 4.0) | ISSN 2525-3409 | DOI: http://dx.doi.org/10.33448/rsd-v10i10.19281

Pereira, A. S., Shitsuka, D. M., Pereira, F. J., \& Shitsuka, R. (2018). Metodologia da Pesquisa Científica (1st ed.). UAB/NTE/UFSM. https://repositorio.ufsm.br/bitstream/handle/1/15824/Lic_Computacao_Metodologia-Pesquisa-Cientifica.pdf?sequence=1\&isAllowed=y

Pinto, J. A., Godoy, L. B. d. M., Marquis, V. W. P. B., Sonego, T. B., Leal, C. d. F. A., \& Ártico, M. S. (2011). Anthropometric data as predictors of Obstructive Sleep Apnea severity. Brazilian Journal of Otorhinolaryngology, 77(4), 516-521. https://doi.org/10.1590/S1808-86942011000400017

Preišegolavičiūtè, E., Leskauskas, D., \& Adomaitienė, V. (2010). Associations of quality of sleep with lifestyle factors and profile of studies among Lithuanian students. Medicina (Kaunas), 482-489. https://doi.org/10.3390/medicina46070070

Quintana, M. I., Gastal, F. L., Jorge, M. R., Miranda, C. T., \& Andreoli, S. B. (2007). Validity and limitations of the Brazilian version of the Composite International Diagnostic Interview (CIDI 2.1). Brazilian Journal of Psychiatry, 29(1), 18-22. https://doi.org/10.1590/S1516-44462006005000024

R Core Team (2020). R: A language and environment for statistical computing. R Foundation for Statistical Computing, Vienna, Austria. URL https://www.Rproject.org/.

Ribeiro, C. R. F., Silva, Y. M. G. P. d., \& Oliveira, S. M. C. d. (2014, April 12). O impacto da qualidade do sono na formação médica. Revista Sociedade Brasileira de Clínica Médica, 12(1). http://www.sbcm.org.br/ojs3/index.php/rsbcm/article/view/45/43

Saygin, M., Öztürk, O., Gonca , T., Has, M., Hayri, U. B., Kurt, Y., Yağlı, M. A., Çalışkan, S., Akkaya, A., \& Öztürk, M. (2016). Investigation of Sleep Quality and Sleep Disorders in Students of Medicine. Turkish Thoracic Journal, 17(4), 132-140. https://doi.org/10.5578/ttj.30513

Vagiakis, E., Kapsimalis, F., Lagogianni, I., Perraki, H., Minaritzoglou, A., Alexandropoulou, K., Rousso, C., \& Kryger, M. (2006, August). Gender differences on polysomnographic findings in Greek subjects with obstructive sleep apnea syndrome. Sleep Medicine, 7(5), 424-430. https://doi.org/10.1016/j.sleep.2005.12.014

Vaz, A.P., Drummond, M., Caetano Mota, P., Severo, M., Almeida, J., \& Winck, J. Carlos (2011). Tradução do Questionário de Berlim para língua Portuguesa e sua aplicação na identificação da SAOS numa consulta de patologia respiratória do sono. Revista Portuguesa de Pneumologia, 17(2),59-65. ISSN: 08732159. Disponível em: https://www.redalyc.org/articulo.oa?id=169722524004 